\title{
Neuropsychological functioning in late-life depression
}

\section{Gro Strømnes Dybedal ${ }^{1}{ }^{*}$, Lars Tanum ${ }^{2}$, Kjetil Sundet ${ }^{3}$, Torfinn Lødøen Gaarden ${ }^{1}$ and Tor Magne Bjølseth ${ }^{1}$}

${ }^{1}$ Department of Geriatric Psychiatry, Diakonhjemmet Hospital, Oslo, Norway

2 Department of Research and Development in Mental Health, Akershus University Hospital, Lørenskog, Norway

${ }^{3}$ Department of Psychology, University of Oslo, Oslo, Norway

\author{
Edited by: \\ Marit Therese Schmid, University of \\ Bergen, Norway \\ Reviewed by: \\ Hilde Katrin Ryland, University of \\ Bergen, Norway \\ Steinunn Adolfsdottir, University of \\ Bergen, Norway \\ *Correspondence: \\ Gro Stromnes Dybedal, Department \\ of Geriatric Psychiatry, \\ Diakonhjemmet Hospital, Pastor \\ Fangens vei 18, 0854 Oslo, Norway \\ e-mail: grostromnes.dybedal@ \\ diakonsyk.no
}

Background: The literature describing neurocognitive function in patients with late-life depression (LLD) show inconsistent findings in regard to incidence and main deficits. Reduced information processing speed is in some studies found to explain deficits in higher order cognitive function, while other studies report specific deficits in memory and executive function. Our aim was to determine the characteristics of neuropsychological functioning in non-demented LLD patients.

Methods: A comprehensive neuropsychological battery was administered to a group of hospitalized LLD patients and healthy control $(\mathrm{HC})$ subjects. Thirty-nine patients without dementia, 60 years or older meeting DSM-IV criteria for current episode of major depression, and 18 non-depressed control subjects were included. The patient group was characterized by having a long lasting current depressive episode of late-onset depression and by being non-responders to treatment with antidepressants. Neurocognitive scores were calculated for the domains of information processing speed, verbal memory, visuospatial memory, executive function, and language. Number of impairments (performance below the 10th percentile of the control group per domain) for each participant was calculated.

Results: Nearly half of the patients had a clinically significant cognitive impairment in at least one neurocognitive domain. Relative to HC subjects, LLD patients performed significantly poorer in the domains of information processing speed and executive function. Executive abilities were most frequently impaired in the patient group (39\% of the patients). Even when controlling for differences in processing speed, patients showed more executive deficits than controls.

Conclusions: Controlling for processing speed, patients still showed impaired executive function compared to HCs. Reduced executive function thus appears to be the core neurocognitive deficit in LLD. Executive function seems to be an umbrella concept for several connected but distinct cognitive functions. Further studies of neuropsychological functioning in LLD patients are needed to characterize more specific what kinds of executive impairments patients have. Additional studies of remitted LLD patients are needed to separate episode-related and persistent impairments.

Keywords: late-life depression, executive function, memory, information processing speed, neuropsychological

\section{NEUROPSYCHOLOGICAL FUNCTIONING IN LATE-LIFE DEPRESSION}

Late-life depression (LLD) refers to the presence of a significant clinical depression in individuals over 60 years of age and is typically defined independently of age at onset (O'Hara et al., 2006). When studying neurocognition in major depression, elderly patients should be identified as a separate group because their age makes them psychobiologically different from younger individuals (Bryan and Luszcz, 2000). Neurocognition in elderly non-demented patients with major depression is characterized by considerable heterogeneity. According to Butters et al. (2004) and Bhalla et al. (2009) about $40-60 \%$ of non-demented patients with late life depression can be classified as cognitively impaired after thorough neuropsychological assessment. However, a considerable number of LLD patients show no significant sign of cognitive impairment. Cognitive deficits tend to persist in the remitted state (Bhalla et al., 2006; Köhler et al., 2010), although in a subgroup of depressed elderly patients with cognitive dysfunction, cognition may improve somewhat in remitters (Butters et al., 2000).

Persistent cognitive deficits after remission in patients with LLD may be related to neurobiological changes, including brain atrophy and an increased prevalence of white matter hyperintensities (Wilkins et al., 2009). There is also increasing evidence for a link between LLD and development of dementia, included Alzheimer disease (Steffens et al., 2006). Cognitive deficits in LLD have been associated with increased rates of relapse 
of depression, disability and poorer response to antidepressant treatment (O'Hara et al., 2006).

The characteristic cognitive profile of this group of patients and possible mechanisms causing impairment, are topics of discussion in the literature. In a recent review, Herrmann et al. (2007) reports that a large proportion of LLD patients suffer from reduced executive function, processing speed, episodic memory, and semantic memory. Visuospatial ability, attention and inhibition, working memory and expressive language have also been observed to be impaired in patients with LLD (O'Hara et al., 2006). Executive dysfunction has systematically been listed as the most pronounced deficit among LLD patients (Herrmann et al., 2007). These findings support the theory put forth by Alexopoulos et al. (2000) stating that frontostriatal dysfunction contributes both to the development of LLD and to executive dysfunction. Differences between LLD patients with early and late onset are frequently not found (Butters et al., 2004; Sheline et al., 2006).

Although frequently referred to as a unitary function (Alexopoulos et al., 2000; Butters et al., 2004), executive function seems to be an umbrella concept for several connected but distinct higher cognitive functions (Miyake et al., 2000a; Hull et al., 2008). In accordance with advices to clinicians from Miyake et al. (2000b), we have made a composite measure of executive function to be used in our analysis in order to reduce some of the weaknesses in reliability and validity of individual measures. We have chosen to classify verbal fluency tests as a language domain. Verbal fluency tests are often listed within the executive function domain, but have been reported as rather insensitive measures of executive function (Henry and Crawford, 2005; Rodriguez-Aranda and Sundet, 2006), and are thus treated separately in our study.

Butters et al. (2004) maintain that reduced information processing is responsible for more specific neuropsychological deficits in LLD, including executive dysfunction. Sheline et al. (2006) also found that slowed processing speed appeared to be the core deficit in LLD. Nebes et al. (2000) noted that reductions in processing resources (speed and working memory) appeared to persist following remission of depression and that these kind of deficits may be trait markers for LLD. Other cognitive deficits could be explained by reductions in processing resources. Sexton et al. (2012) concludes that impairments in executive function or processing speed were sufficient to explain differences in episodic memory and language skills in their group of (remitted) LLD patients. They also found that executive deficits could not be fully explained by impairments in processing speed.

Our aims are (1) to analyse and describe neuropsychological function in LLD patients, and to compare this group with healthy controls (HCs). We will focus on the domains of information processing speed, memory, verbal fluency, and executive function. Our hypothesis is that depressed patients are characterized by cognitive deficits in all of these domains, but have the most pronounced deficits in information processing speed and executive function. (2) We want to examine the incidence of cognitive impairment in LLD patients by calculating the number of impaired cases in the patient group compared to the control group. (3) We also want to investigate if a general deficit, like information processing speed slowing, is a main deficit that can explain deficits in other more specific cognitive domains, including memory and executive function.

\section{MATERIALS AND METHODS PARTICIPANTS}

Norwegian speaking Caucasian inpatients meeting the DSM-IV criteria of major depression (single episode, recurring depression or bipolar disease) during the period 01.09.09 to 20.12.12 were asked to join the study, unless they met the exclusion criteria. The study was approved by the Regional Committee for Research Ethics (REK) in Norway which included an approval of the use of ECT as a first-line medical treatment in selected cases. In this paper we present baseline data from testing of a group of inpatients that some days after testing was given ECT. They were also tested after ECT-series and after an additional 3 months. Inclusion criteria were age 60-85 years old, being hospitalized in the Department of Geriatric Psychiatry (DGP), Diakonhjemmet Hospital, and providing a written consent to participate in baseline testing and later randomization to bifrontal or unilateral electrode placement in electroconvulsive therapy (ECT). Patients were excluded if (1) having a Mini Mental State Examination (MMSE) Score (Folstein et al., 1975) of less than 24 (maximum 30 points), as this could be a strong indication of dementia, (2) a diagnosis of dementia or other neurodegenerative disorder within the follow-up period of 5 months. Patients with clear signs of cognitive impairment at baseline, were followed especially closely and underwent an interdisciplinary dementia assessment, (3) other diagnosis of neurological disorder; e.g., head injury, stroke or Mb. Parkinson, (4) current or earlier substance abuse, (5) rapid cycling bipolar disorder, (6) schizophrenia or schizoaffective disorder. Our criteria for participation in the study allowed patients with mild cognitive impairment (either amnestic or other type) to be included in the study, because older adults with depression often present with signs and symptoms indicative of cognitive impairment (Wilkins et al., 2009). (7) Since the patients were given ECT-treatment after baseline-testing, there were some exclusion criteria related to this kind of treatment, e.g., that ECT has not had any effect in an earlier depressive episode or ECT treatment during the last 6 months. In order to relate cognitive function of LLD patients to an age-matched group without a history of psychiatric illness, we recruited 20 elderly patients from a recreational center in the community. Eighteen of these patients had a MMSE score of 24 or better at baseline and were included as controls.

\section{CLINICAL ASSESSMENT}

Clinical assessment at admission was carried out by a trained psychiatrist (TMB or TLG) who also scored Hamilton Depression Scale (17-items) (HAM-D 17, Hamilton, 1960). TMB also assessed all the patients with MINI International Neuropsychiatric Interview; specifically the MINI-Plus (Sheehan et al., 1998) before inclusion. He had participated in a structured training program for MINI-Plus. Patients with a higher score than 17 points on HAM-D 17 and diagnosis of major depression according to MINI could be included. Age at onset, number of previous episodes, other disease parameters and somatic morbidity were determined by the psychiatrist (TMB or TLG) 
from the clinical interview. A thorough examination of medical records and scoring of Cumulative Illness Rating Scale for Geriatric Patients (CIRS-G) (Miller et al., 1992) was also performed. For our purposes, the psychiatric item of the CIRS-G was excluded. The psychiatrist (TMB) also administered HAM-D 17 and MMSE to controls, evaluated their somatic morbidity with CIRS-G and ascertained that they did not have a history of any major psychiatric illness.

\section{NEUROCOGNITIVE ASSESSMENT}

Neurocognitive assessment of the patients and controls were carried out by the clinical neuropsychologist (GSD) or a nurse. The nurse had been trained by GSD in administering the test battery. Another trained nurse and an occupational therapist had received training by GSD and they assisted with testing of the HCs. The test battery was chosen with the main rationale of giving measurements of memory and executive function as a background for assessing cognitive adverse effects of ECT. Wanting patients to be motivated to go through testing three times within a period of about 5 months, we composed a test battery that was comprehensive, but not too strenuous, consisting of subtests from standard test batteries validated for Norwegian use. Learning and memory was measured using the official Norwegian research version of the Hopkins verbal learning test-revised (HVLT-R, Brandt and Benedict, 2001), and the Brief Visuospatial Memory testR (BVMT-R, Benedict, 1997). Information processing speed was assessed by the Trail Making test part A (Reitan and Wolfson, 1993) and D-KEFS Color Word Interference test part 1 and 2 (CWIT, Delis et al., 2005). Aspects of executive function were assessed by D-KEFS Tower Test (Delis et al., 2005), the CWIT part 3 and 4 (Delis et al., 2005) and the Trail Making test part B (Reitan and Wolfson, 1993). The CWIT part 3 is based on the Stroop (1935) procedure. Verbal fluency was assessed with the letter fluency from the D-KEFS battery (Delis et al., 2005) and the animal naming test (Borowski et al., 1967). The subtest Vocabulary from the Wechsler Abbreviated Scale of intelligence (WASI) (Wechsler, 2007) was used as an estimate of premorbid intelligence. Global cognitive function was assessed by a revised version of the MMSE by Engedal et al. (1988). Patients who did not manage the serial subtraction by sevens in MMSE were not tested with backward spelling, which was assumed to be a more easy measure of attention (Ganguli et al., 1990). A selfconstructed unpublished "Media questionnaire" consisting of 20 questions was intended for repeated assessments studying retrograde amnesia for information presented in the news prior to treatment (2003-2008). The questionnaire served also as a measure of general public knowledge among patients and controls. An adapted version of the Autobiographical Memory InterviewShort Form (AMI-SF, McElhiney et al., 1997) measuring personal semantic memories was administered. It is designed for repeated use after ECT-treatments and measures retrograde amnesia for autobiographical memories. It is commonly used in ECT-studies, but has lately been criticized because of weak separation between normal forgetting over time and retrograde amnesia and lack of validiation studies (Semkovska and McLoughlin, 2013). We adapted AMI-SF for older subjects before the start of the study. After data from the control subjects were collected, we deleted five items that could not be answered consistently 2 months after baseline by a majority of healthy elderly. These items seemed to threaten the validity of AMI-SF as a measure of retrograde amnesia. Maximum score in our adapted version is 30 compared to 60 in the original McElhiney et al. (1997) version.

There were little missing data, except for The Trail Making test part B that was too difficult for 8 patients who gave up before the task was completed. Results are also missing for two controls on this test. One patient and two controls did not complete the CWIT part 1 and 2, three patients and two controls did not complete part 3 and six patients and two controls did not complete part 4 . There are missing scores on the Tower Test for one patient and one control. In addition, there is one missing score for controls on the Trail Making Test part A and one missing score for patients on the Animal Naming Test and the Letter Fluency Test.

Our test battery was organized into 5 domains based mainly on the current knowledge about what each test measures (Strauss et al., 2006; Lezak et al., 2012). Raw scores were transformed into $z$-scores, using the means and standard deviations of the elderly control sample. The variance in the control group's scores was checked and we found no major threat to validity of the transformed $z$-scores. Missing scores were replaced with the average score for the group (patient or control). The $z$-scores were then averaged within each neuropsychological area to produce domain scores. The domain Information processing speed consists of the CWIT part 1 and 2 and the Trail Making test part A. The domain of Verbal memory consists of 3 scores from the HVLT-R; total learning, delayed recall and discrimination (recognition). The domain of visuospatial memory consists of three scores from the BVMT-R; total learning, delayed recall and discrimination. The domain of language consists of scores from two fluency tests; the animal learning test and the letter fluency. The executive domain consists of the Trail Making test part B, the DKEFS Tower test and the CWIT part 3 (time + errors divided by two). The CWIT part 3 time and the CWIT part 3 errors were highly correlated $(r=0.486, p<0.001)$, supporting that the measures are related, although they measure different aspects of performance. We chose to exclude part 4 of the CWIT from our analyses. The CWIT 4 was designed to be the most difficult part of the test (Delis et al., 2005). But several studies question the validity of the CWIT 4 as an equally or more sensitive test of executive impairment than the CWIT 3 in clinical groups (Lippa and Davis, 2010; Savla et al., 2011). In addition, several patients in our study did not comply with the CWIT 4 at the 3 months follow-up, although they did at baseline. Including the CWIT 4 within the baseline domain score, would leave us with an executive function score that could not be used at the follow-up. Another reason to exclude the CWIT 4 was more missing scores at baseline. The CWIT 3 time and the CWIT 4 time scores were highly correlated $(r=0.464, p=0.001)$, supporting the expectation (Delis et al., 2005) that they partly measure the same executive function (inhibition). Chronbach's alpha and mean inter item correlation for domain scores were: Information processing domain $(\alpha=0.72 / r=0.48)$, Verbal memory $(\alpha=0.85 / r=$ $0.65)$, Visuospatial memory $(\alpha=0.86 / r=0.70)$, Executive function $(\alpha=0.73 / r=0.51)$, and Language $(\alpha=0.42 / r=$ $0.27)$. The number of domains $(0-5)$ in which each subject was 
impaired (defined as performing below the 10th percentile of the control group) was calculated for both groups.

\section{STATISTICAL ANALYSIS}

The Statistical Package for the Social Sciences (SPSS Inc., Chicago, IL; version 20.0) was used. Means and standard deviations are reported for continuous variables and percentages for categorical variables. Clinical data were statistically analyzed using conventional descriptive methods and $t$-tests. Statistical significance was determined using the 0.05 level and 2-tailed tests of significance. Dichotomous variables were analyzed with crosstabs with corresponding post hoc analyses performed using chi-squares. In situations where the expected cell frequencies were $<5$, Fisher's exact test was used. Correlation analyses (Spearman) explored whether neurocognition was associated with demographic variables or clinical variables. One-way ANCOVAs with age as a covariate were used to compare the groups on continuous variables. Multivariate tests were not used on neurocognitive raw scores because of missing data, especially on the Trail Making test. The relative impact on neurocognition by diagnosis of LLD was investigated by a one way MANCOVA with all 5 neurocognitive domain scores entered as dependent variables. Age was entered as a covariate. Then, a MANCOVA with information processing speed and age as covariates was conducted, investigating whether variability in neurocognitive function would then be explained by reduced processing speed. We followed-up significant findings in the MANCOVAs by single-test analysis of covariance. Effect sizes are reported as partial eta squared. Bonferroni corrections were performed by dividing the p-value by number of tests/domains. Lastly, a comparison of number of cognitive impairments in depressed patients and controls was done with a Mann-Whitney $U$-test.

\section{RESULTS}

\section{CLINICAL CHARACTERISTICS OF THE PATIENT GROUP}

Demographic and clinical characteristics of the LLD and HC groups are presented in Table 1 . There were no statistically significant group differences between the LLD and HC participants regarding age, education, gender, estimated premorbid IQ (Vocabulary, WASI), global cognitive function (MMSE), total medical disease burden (CIRS-G) or vascular burden (CIRS$\mathrm{G}$ heart and vascular score). There was a 3 year age difference between groups ( $p=0.087$ ) which may represent a possible confounder for neurocognitive performance. Group comparisons were therefore performed with age as covariate.

The majority of patients had a severe unipolar depression without psychosis, but with somatic syndrome. The mean age of first depression episode was in the mid-fifties. As a group, the patients had previously rather few life-time depressive episodes and few previous hospitalizations, but reported a long mean duration of the current episode. $46.1 \%$ of the patients could be considered as medication-resistant defined as no clinical response

Table 1 | Characteristics of the study participants.

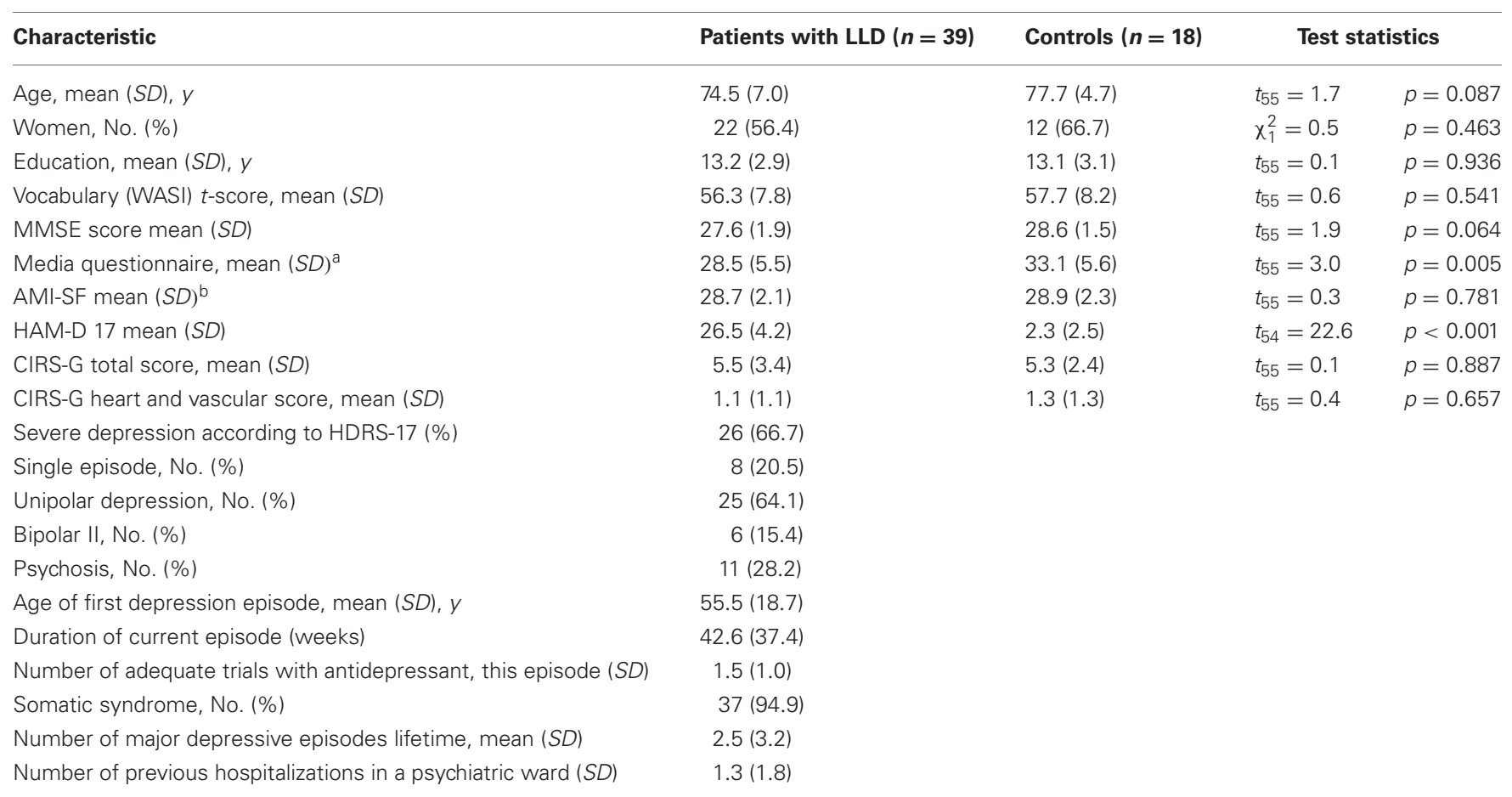

Abbreviations: AMI-SF, Autobiographical Memory Interview-short form; CIRS-G, Cumulative IIIness Rating Scale-Geriatrics; HAM-D 17, Hamilton Depression Rating Scale-17 items; LLD, late-life depression; MMSE, Mini Mental State Examination; WASI, Wechsler Abbreviated Scale of Intelligence.

${ }^{a}$ Self-constructed, unpublished. Maximum score is 40 .

${ }^{b}$ Modified for use in this study. Maximum score is 30. 
to previous treatment with at least two different antidepressive agents given in adequate doses over an adequate period of time. An additional $38.5 \%$ had no clinical response to previous treatment with one antidepressive agent given in adequate doses over at least 4 weeks.

\section{NEUROCOGNITIVE PERFORMANCE}

Table 2 presents raw scores on neuropsychological tests. Using age as a covariate in the statistical analyses of group differences, Table 2 shows that the differences were reaching the level of nominal significance in six of the 15 single measures. One of the single measures (Trail Making test part B) remained statistically significant after Bonferroni corrections (setting the $p$-value at 0.003). A more detailed analysis of performance on the Tower test showed that the number of correct solved towers were significantly different between the groups $\left[F_{(1,52)}=10.63, p=0.002\right]$. There were no significant differences in how effective patients and controls were in solving the tower tasks they eventually succeeded with (moves used on tasks solved / minimum moves needed to solve these tasks) [Mean efficacy of patients $1.2(0.3)$ and controls 1.23 $\left.(0.2), F_{(1,49)}=1.5, p=0.229\right]$.

Table 3 presents correlations between the raw scores and demographic variables for the patient group. There were several significant correlations between age and neurocognitive measures, confirming the benefit of using age as a covariate in further analysis. Correlations between cognitive measures and depression score show small to medium effects. Sex and education levels have modest effects on some tests, while somatic morbidity shows very small effects.

A one-way between-groups multivariate analysis of variance was performed to investigate group differences in cognitive function, by domains. The results are shown in Table 4. Five dependent variables were used; the domain scores Information processing speed, Verbal memory, Visuospatial memory, Executive function, and Language. The independent variable was group (patient vs. controls), age was covariate. There was a statistically significant difference between LLD patients and controls on the combined dependent variables $\left[F_{(5,50)}=3.7\right.$, $\left.p=0.006, \eta_{p}^{2}=0.27\right]$. When analyzing domain scores separately, Information processing and Executive function reached statistical significance, using an alpha level of 0.05 , with LLD patients performing poorer than controls. After Bonferroni corrections the executive function domain remains significant.

A second one-way between-groups multivariate analysis of variance with Information processing speed and age as covariates was performed for the four domain scores Verbal memory, Visuospatial memory, Executive function, and Language. The difference between LLD patients and controls remained statistically

Table 2 | Results of ANCOVA tests comparing LLD patients' and Control subjects' raw score performance on individual neurocognitive tests*a

\begin{tabular}{|c|c|c|c|c|c|}
\hline Test & Patients $(n=39)$ & Controls $(n=18)$ & \multicolumn{2}{|c|}{ ANCOVA $^{a}$} & $\eta_{p}^{2}$ \\
\hline C-W Interference—color naming ${ }^{b}$ & $41.9(10.5)$ & $35.3(6.3)$ & 7.1 & 0.011 & 0.12 \\
\hline C-W Interference-word reading ${ }^{b}$ & $27.5(6.1)$ & $27.6(5.6)$ & 0.0 & 0.838 & 0.001 \\
\hline Trail Making test A & $63.4(21.6)$ & $53.1(17.9)$ & 5.2 & 0.027 & 0.09 \\
\hline HVLT-R total learning & $21.1(4.7)$ & $21.9(5.7)$ & 1.6 & 0.218 & 0.03 \\
\hline HVLT-R delayed recall & $7.1(2.7)$ & $7.5(2.4)$ & 1.2 & 0.282 & 0.02 \\
\hline HVLT-R discrimination ${ }^{c}$ & $10.7(1.3)$ & $10.8(1.3)$ & 0.1 & 0.714 & 0.003 \\
\hline \multicolumn{6}{|l|}{ VISUOSPATIAL MEMORY } \\
\hline BVMT-R total learning & $12.5(5.7)$ & $14.4(6.9)$ & 2.9 & 0.097 & 0.05 \\
\hline BVMT-R delayed recall & $4.8(2.7)$ & $5.4(2.5)$ & 1.9 & 0.180 & 0.03 \\
\hline Trail Making test $\mathrm{B}$ & $191.5(78.4)$ & $139.4(61.0)$ & 10.0 & 0.003 & 0.19 \\
\hline C-W Interference-inhibition, time ${ }^{b}$ & $100.7(33.3)$ & $82.8(22.9)$ & 5.6 & 0.022 & 0.10 \\
\hline C-W Interference-inhibition errors ${ }^{b}$ & $3.4(4.4)$ & $0.9(1.1)$ & 6.6 & 0.013 & 0.12 \\
\hline \multicolumn{6}{|l|}{ LANGUAGE } \\
\hline Animal naming test & $17.3(5.5)$ & $18.7(6.1)$ & 2.8 & 0.103 & 0.05 \\
\hline Letter fluency $(F, A, S)^{b}$ & $38.4(12.3)$ & $39.2(11.4)$ & 0.01 & 0.931 & $<0.001$ \\
\hline
\end{tabular}

Abbreviations: BVMT-R, Brief Visuospatial Memory Test-revised; C-W interference, Color Word Interference Test (variant of the Stroop test); HVLT-R, Hopkins verbal learning test-revised.

${ }^{*}$ Data are given as mean (SD) of conventional scoring method.

${ }^{a}$ Age is used as a covariate.

${ }^{b}$ D-KEFS, Delis Kaplan Executive Function System.

${ }^{c}$ Recognition; number correct minus false positives. 
Table 3 | Pearson correlations between demographical variables and the patients' neuropsychological raw scores ${ }^{\mathrm{a}}$.

\begin{tabular}{|c|c|c|c|c|c|}
\hline Neuropsychological test & Sex & Age & Education & CIRS-Gb & Depression ${ }^{\mathrm{C}}$ \\
\hline HVLT-R delayed recall & 0.08 & -0.25 & 0.05 & 0.04 & -0.26 \\
\hline BVMT-R total learning & $0.39 *$ & $-0.36^{*}$ & 0.26 & -0.02 & -0.26 \\
\hline BVMT-R delayed recall & $0.33^{*}$ & $-0.39 *$ & $0.35 *$ & -0.04 & -0.20 \\
\hline BVMT-R discrimination & -0.09 & -0.28 & 0.10 & -0.09 & -0.21 \\
\hline C-W Interference—color naming ${ }^{d}$ & 0.04 & 0.16 & 0.24 & 0.03 & 0.20 \\
\hline C-W Interference-word reading ${ }^{d}$ & 0.04 & 0.15 & 0.04 & 0.11 & -0.21 \\
\hline C-W Interference-inhibition, time ${ }^{d}$ & -0.001 & 0.30 & -0.30 & -0.18 & 0.10 \\
\hline C-W Interference-inhibition, errors ${ }^{d}$ & -0.21 & 0.23 & -0.17 & -0.15 & 0.12 \\
\hline Animal naming test & 0.12 & $-0.37^{*}$ & -0.07 & -0.08 & -0.08 \\
\hline Letter fluency $(F, A, S)^{d}$ & 0.28 & 0.22 & $0.33^{*}$ & 0.25 & -0.01 \\
\hline
\end{tabular}

Abbreviations: BVMT-R, Brief Visuospatial Memory Tes-revised; C-W Interference, Color-Word Interference test (variant of the Stroop test); CIRS-G, Cumulative IIIness Rating Scale-Geriatrics; HVLT-R, Hopkins verbal learning test-revised.

${ }^{a}$ Results are given for patients with $L L D(n=39)$.

${ }^{b}$ Total score.

${ }^{c}$ HAM-D 17, Hamilton Depression Rating Scale-17 items.

${ }^{d} D-K E F S$, Delis Kaplan Function System.

${ }^{*} P<0.05 ;{ }^{* *} P \leq 0.01$.

Bold values indicate the correlation between HVLT total learning and depression is -0.34 and significant.

Table 4 | Results on MANCOVA tests comparing elderly control subjects' and LLD patients' z-score performance on cognitive domains $^{\mathrm{a}}$.

\begin{tabular}{lllll}
\hline Domain & Mean (SD) & $\mathbf{F}_{(\mathbf{1}, \mathbf{5 4})}$ & $\boldsymbol{p}$ & $\boldsymbol{\eta}_{\mathbf{p}}$ \\
\hline Information Processing Speed $^{\mathrm{b}}$ & $-0.5(1.0)$ & 5.9 & 0.019 & 0.10 \\
Verbal Memoryc $^{\mathrm{c}}$ & $-0.1(0.9)$ & 1.0 & 0.318 & 0.02 \\
Visuospatial Memory $^{\mathrm{d}}$ & $-0.3(0.9)$ & 3.0 & 0.087 & 0.05 \\
Executive Functione $^{\mathrm{e}}$ & $-1.1(1.2)$ & 17.4 & $<0.001$ & 0.24 \\
Language $^{f}$ & $-0.15(0.8)$ & 0.9 & 0.338 & 0.02
\end{tabular}

Abbreviations: $L L D$, late-life depression.

${ }^{a}$ Results are given for the patients with $L L D(n=39)$, age is used as a covariate.

${ }^{b}$ Trail Making test A, D-KEFS C-W Interference test color naming and word reading (average score).

${ }^{c}$ Three measures from HVLT-R (average score).

${ }^{d}$ Three measures from BVMT-R (average score).

${ }^{e}$ Trail Making test B, D-KEFS Tower test, and (C-W interference-inhibition, time $+C$-W interference, inhibition, errors)/2 (average score).

${ }^{f}$ Animal naming test and D-KEFS Letter Fluency $(F, A, S)$.

significant on the combined dependent variables, $F_{(4,50)}=2.9$, $p=0.030, \eta_{p}^{2}=0.19$, as well as the Executive function when conducted separately $\left[F_{(1,53)}=10.4, p=0.002\right]$. After Bonferroni corrections the executive function domain remains significant.

Investigating the number of impaired cases, we found that the depressed patients were impaired in more domains than control subjects (Mann-Whitney $U$-test, $p=0.05$ ). Table 5 shows that in the patient group, close to $50 \%$ of the patients experienced a

\begin{tabular}{|c|c|c|}
\hline No. of deficits & $\begin{array}{l}\text { Patients with LLD, \% } \\
\text { ( } \mathrm{n}=39)\end{array}$ & $\begin{array}{l}\text { Controls, } \% \\
\text { ( } \mathrm{n}=18)\end{array}$ \\
\hline 0 & 51.3 & 77.8 \\
\hline 1 & 20.5 & 11.1 \\
\hline 2 & 15.4 & 11.1 \\
\hline 3 & 10.3 & 0 \\
\hline 4 & 0 & 0 \\
\hline 5 & 2.6 & 0 \\
\hline Total & 100 & 100 \\
\hline Mean No. of deficits (SD) & $1.0(1.2)$ & $0.3(0.7)$ \\
\hline
\end{tabular}

Abbreviation: $L L D$, late-life depression.

clinically significant impairment in at least one cognitive domain compared to approximately $20 \%$ of the controls. Figure 1 shows performance on cognitive domains by LLD patients and controls. More depressed patients were impaired in the executive domain (39 vs. $5.6 \%$ of controls, $\chi^{2}=6.6, p=0.010$ ), but the differences did not reach significance in the other domains.

\section{DISCUSSION}

Patients with late-life major depression were characterized by slower information processing speed and a markedly stronger degree of executive deficits than non-depressed HCs. When controlling for information processing speed, the difference in the 


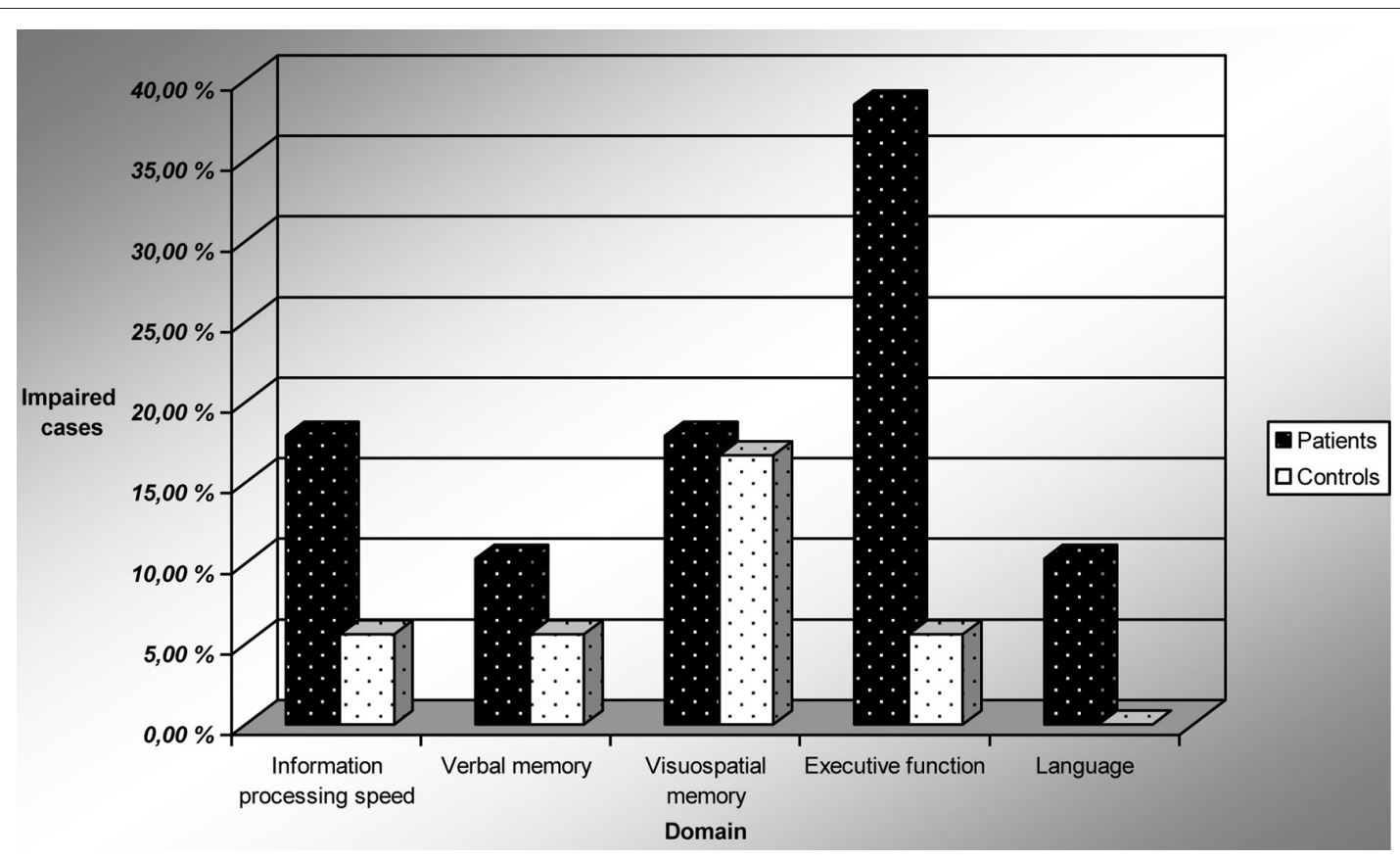

FIGURE 1 | Performance on cognitive domains by LLD patients and controls. Impairment is defined as performance below the 10 th percentile of the control subjects. Abbreviation: LLD, late life depression.

domain of executive function remained significant. There were no statistically significant differences in verbal memory, visuospatial memory or language (verbal fluency) between groups. Our findings are consistent with others reporting main differences between such groups on tests of information processing speed and executive function (Herrmann et al., 2007).

Contrary to the findings reported by Butters et al. (2004), but in line with Sexton et al. (2012) and Sheline et al. (2006), we found that deficits in the executive function could not be fully explained by a general information processing speed deficit. Such differences between study reports may be partly explained by the composition of the test battery and how the composite neurocognitive measures are defined. Even more important is probably differences between patient groups. The main features of our inpatient group seem to be different from the outpatient group of Butters et al. (2004). They studied mainly outpatients not yet prescribed antidepressants at the time of testing and $50 \%$ of the patients experienced their first depressive episode. The LLD patients in our study were characterized by among other factors vegetative symptoms, non-responding to treatment with antidepressants, and a protracted course of current depressive episode. Non-remitters on antidepressants more often show executive deficits (Steffens and Potter, 2008; Snyder, 2013).

Consistent with the results of Meeter et al. (2011) the patients' scores on a test that assessed memory for news were lower than those of controls (Media Questionnaire). In accordance with Butters et al. (2004), but in contrast to O'Brien et al. (2004), we did not find significant differences between groups on tests of verbal fluency. We did not find any significant difference between the groups in the memory domain either, in contrast to what has been reported by others (Butters et al., 2004; Köhler et al., 2010;
Mesholam-Gately et al., 2012). Our results underline that neurocognitive impairment in non-demented LLD patients does not necessarily include memory impairment. Given that one out of several common pathways linking LLD, mild cognitive impairment and at a later stage dementia, may be cerebrovascular disease and Alzheimer neuropathology (Butters et al., 2008), it is natural to expect memory impairment at least in subgroups of nondemented LLD patients. A likely explanation of different results within the memory domain, are differences in patient and control populations and in inclusion and exclusion criteria. Butters et al. (2004) state that they were "using minimal exclusionary criteria to maximize the heterogeneity among LLD patients, thus permitting evaluation of a range of potential risk factors for cognitive impairment" (p. 593). Our choice of exclusionary criteria appears to have been more comprehensive.

In our study, significantly more depressed patients were cognitively impaired than $\mathrm{HC}$ subjects. More depressed patients had executive deficits, but the differences did not reach statistical significance in the domains of verbal memory, visuospatial memory, information processing speed and language. In our study $51.3 \%$ of the LLD patients were unimpaired, vs. $77.8 \%$ of the controls. In comparison, Butters et al. (2004) found that $39 \%$ of their LLD patients and $67.5 \%$ of their controls were unimpaired. Common for the studies is that a substantial part of LLD patients have cognitive deficits.

The largest effect sizes when comparing LLD patients and controls were seen on the Tower test and the Trail Making test part B. These tests are speed-dependent. But in addition they require executive abilities like mental flexibility, planning and working memory. Several studies have found that LLD patients have lower scores on TMT B (e.g., Lockwood et al., 2002; 
O'Brien et al., 2004). Our findings are conservative because we choose to record lacking capability to carry through the test as a missing score and not maximum score.

Mood disorders, and in particular depressive disorders, have been believed to impact overall executive functioning. The Tower test is regarded as a sensitive test of this domain, but very few studies have actually examined the effects of mood disorders on Tower performance (Sullivan et al., 2009). As shown in our study, the patients were just as effective as controls (total moves/minimum moves) when working on Tower tasks that they solved, but they gave up more quickly and solved fewer items. There was also no difference between groups with regard to rule infractions while performing the test.

\section{CONCLUSIONS}

Non-demented patients with LLD were significantly more cognitively impaired than HCs. Nearly $50 \%$ of patients showed clinically significant impairment (performance below the 10th percentile of the control group) in at least one of five neurocognitive domains. Approximately $40 \%$ of the patients showed deficits in the domain of executive function and nearly $20 \%$ in the domain of information processing speed, but in other cognitive domains they were not significantly more impaired than controls. When Information processing speed is accounted for, patients still show deficits in executive function. The Tower Test and the Trail Making Test part B seem to be especially sensitive tests for detecting cognitive deficits in LLD-patients. Future studies are needed to clarify the exact nature of the executive deficits and the degree of persistence of deficits after successful clinical treatment. Numerous studies have documented the weaker effect of antidepressants in the subgroup of LLD-patients with executive deficits (Story et al., 2008), but there is a lack of studies on the effect of ECT-treatment in this group. There are several clinical implications of our findings. LLD patients ought to be screened routinely for cognitive impairment at admission at a DGP. Results from screening tests and in particular from more detailed neuropsychological assessment can assist therapists in tailoring treatment and follow-up to the patients' individual needs. Realistic expectations and tailored treatment can hopefully make relapse of depression after remission less likely.

\section{STRENGTHS AND LIMITATIONS}

A strength of our study is the exclusion of patients with suspected dementia or other neurological diseases at admission. By following up the patients over 5 months after baseline-testing, we were able to exclude patients who were given a dementia diagnosis during their hospitalization or follow up period.

Our neurocognitive test battery was comprehensive and broad-based, especially in the executive domain. Instead of using just one or two well-known "executive tests," we created a composite measure consistent with the recommendation in Miyake et al. (2000b). Our patient population was comparable to other relevant study populations with regard to education, gender, estimated IQ and medical morbidity.

There are a number of potential limitations. The control group is small and cognitive impairment is not the defined 10\% across domains. It varies from 0 to $18 \%$. On the two domains with evident differences between groups, the percentage of impairment in the $\mathrm{HC}$ group was less than $6 \%$. An additional limitation is that the size of the patient group is also rather small. The Low $N$ in the patient group may cause reduced power to detect real differences. By reporting effect sizes of group differences, we invite the reader to decide whether non-significant findings represent possible effects given larger subject samples. The mean age of the controls was 3 years older than the patients' and may have exaggerated group differences that were age dependent. This threat to external validity was addressed by using age as a covariate variable in group comparisons.

Results from neurocognitive testing could possibly be influenced by the effect of neurotropic medication. This is difficult to avoid when studying a group of in-patients with mainly long-standing symptoms of a depressive episode. The domain of Language showed a rather weak internal consistency. Each test in this domain should perhaps be separately considered in the later analysis of adverse effects of ECT on neurocognition in elderly patients.

\section{ACKNOWLEDGMENTS}

This research project was financially supported by the Norwegian ExtraFoundation for Health and Rehabilitation through EXTRA funds. We would like to thank Diakonhjemmet Hospital for securing good conditions for researchers, including secretary services, and for the means to assess a healthy control group in 2010 . We are also thankful for the valuable supervision from professor in neuropsychology dr. philos. Ivar Reinvang when making a "Media questionnaire." Registered nurse Marianne Larsen offered extensive and skillful assistance with neuropsychological testing of the patients and the healthy controls. The authors would like to thank the staff at department of Geriatric Psychiatry, for their support and engagement in the study.

\author{
REFERENCES \\ Alexopoulos, G. S., Meyers, B. \\ S., Young, R. C., Kalayam, B., \\ Kakuma, T., Gabrielle, M., et al. \\ (2000). Executive dysfunction \\ and long-term outcomes of \\ geriatric depression. Arch. Gen. \\ Psychiatry 57, 285-290. doi: \\ 10.1001/archpsyc.57.3.285 \\ Benedict, R. H. B. (1997). Brief \\ Visuospatial Memory Test-Revised.
}

Lutz, FL: Psychological Assessment Resources, Inc.

Bhalla, R. K., Butters, M. A., Becker, J. T., Houck, P. R., Snitz, B. E., Lopez, O. L., et al. (2009). Patterns of mild cognitive impairment after treatment of depression in the elderly. Am. J. Geriatr. Psychiatry 17, 308-316. doi: 10.1097/JGP.0b013 e318190b8d8
Bhalla, R. K., Butters, M. A., Mulsant, B. H., Begley, A. E., Zmuda, M. D., Schoderbek, B., et al. (2006). Persistence of neuropsychological deficits in the remitted state of late-life depression. Am. J. Geriatr. Psychiatry 14, 419-427. doi: 10.1097/01.JGP.0000203130. 45421.69

Borowski, J. G., Benton, A. L., and Spreen, O. (1967). Word fluency and brain damage. Neuropsychology 5, 135-140. doi: 10.1016/0028-3932(67)90015-2 Brandt, J., and Benedict, R. H. B. (2001). The Hopkins Verbal Learning Test-Revised. Professional Manual. Odessa, FL: Psychological Assessment Resources, Inc.

Bryan, J., and Luszcz, M. A. (2000). Measurement of executive function: considerations for detecting 
adult age differences. J. Clin. Exp. Neuropsychol. 22, 40-55. doi: 10.1076/1380-3395(200002)22:1;18;FT040

Butters, M. A., Becker, J. T., Nebes, R. D., Zmuda, B. H., Pollock, B. G., and Reynolds, D. F. (2000). Changes in cognitive functioning following treatment of late-life depression. Am. J. Psychiatry 157, 1949-1954. doi: $\quad$ 10.1176/appi.ajp.157. 12.1949

Butters, M. A., Young, J. B., Lopez, O., Aizenstein, H. J., Mulsant, B. H., Reynolds, C.F. 3rd., et al. (2008). Pathways linking late-life depression to persistent cognitive impairment and dementia. Dialogues Clin. Neurosci. 10, 345-357.

Butters, M. A., Whyte, E. M., Nebes, R. D., Begley, A. E., Dew, M. A., Mulsant, B. H., et al. (2004). The nature and determinants of neuropsychological functioning in late-life depression. Arch. Gen. Psychiatry 61, 587-595. doi: 10.1001/archpsyc.61. 6.587

Delis, D. C., Kaplan, E., and Kramer, J. H. (2005). Delis-Kaplan Executive Functions System (D-KEFS) Norwegian Manual. Stockholm: Pearson Assessment.

Engedal, K., Haugen, P. K., Gilje, K., and Laake, K. (1988). Efficacy of short mental tests in the detection of mental impairment in old age. Compr. Gerontol. A. 2, 87-93.

Folstein, M. F., Folstein, S. E., and McHugh, P. R. (1975). "Mini Mental State." A practical method of grading the cognitive state of patients for the clinician. J. Psychiatr. Res. 12, 189-198. doi: $\quad 10.1016 / 0022-3956(75)$ 90026-6

Ganguli, M., Ratcliff, G., Huff, F. J., Belle, S., Kancel, M. J., Fischer, L., et al. (1990). Serial sevens versus world backwords: a comparison of the two measures of attention from the MMSE. J. Geriatr. Psychiatry Neurol. 3, 203-207. doi: $\quad 10.1177 / 089198879000$ 300405

Hamilton, M. (1960). A rating scale for depression. J. Neurol. Neurosurg. Psychiatry 23, 56-62. doi: 10.1136/jnnp.23.1.56

Henry, J. D., and Crawford, J. R. (2005). A meta-analytic review of verbal fluency deficits in depression. J. Clin. Exp. Neuropsychol. 27, 78-101. doi: 10.1080/13803399 0513654

Herrmann, L. L., Goodwin, G. M., and Ebmeier, K. P. (2007).
The cognitive neuropsychology of depression in the elderly. Psychol. Med. 37, 1693-1702. doi: $\quad 10.1017 /$ S0033291707 001134

Hull, R., Martin, R. C., Beier, M. E., Lane, D., and Hamilton, A. C. (2008). Executive function in older adults: a structural equation modelling approach. Neuropsychology 22, 508-522. doi: $\quad 10.1037 / 0894-4105.22$. 4.508

Köhler, S., Thomas, A. J., Barnett, N. A., and O'Brien, J. T. (2010). The pattern and course of cognitive impairment in late-life depression. Psychol. Med. 40, 591-602. doi: $\quad 10.1017 /$ S00332917099 90833

Lezak, M. D., Howieson, D. B., Bigler, E. D., and Tranel, D. (2012). Neuropsychological Assessment. 5th Edn., New York, NY: Oxford University Press.

Lippa, S. M., and Davis, R. N. (2010). Inhibition / switching is not necessarily harder than inhibition: an analysis of the D-KEFS color word interference test. Arch. Clin. Neuropsychol. 25, 146-152. doi: 10.1093/arclin/ acq001

Lockwood, K. A., Alexopoulos, G. S., and van Gorp, W. G. (2002). Executive dysfunction in geriatric depression. Am. J. Psychiatry 159, 1119-1126. doi: 10.1176/appi.ajp.159.7.1119

McElhiney, M., Moody, B., and Sackeim, H. (1997). The Autobiographical Memory Interview - Short Form. New York, NY: New York State Psychiatric Institute.

Meeter, M., Murre, J. M. J., Janssen, S. M. J., Birkenhager, T., and van Den Broek, W. W. (2011). Retrograde amnesia after electroconvulsive therapy: a temporary effect. J. Affect. Disord. 132, 216-222. doi: 10.1016/j.jad.2011. 02.026

Mesholam-Gately, R., Giuliano, A. J., Zillmer, E. A., Barakat, L. P., Kumar, A., Gur, R. C., et al. (2012). Verbal learning and memory in older adults with minor and major depression. Arch. Clin. Neuropsychol. 27, 196-207. doi: 10.1093/arclin/acr 106

Miller, M. D., Paradis, C. F., Houck, P. R., Mazumdar, S., Stack, J. A., Rifai, A. H., et al. (1992). Rating chronic illness burden in geropsychiatric practice and research: application of the cumulative illness rating scale. Psychiatry Res. 41, 237-248. doi: $\quad 10.1016 / 0165-1781(92)$ 90005-N
Miyake, A., Friedman, N. P., Emerson, M. J., Witzki, A. H., Howerter, A., and Wager, T. D. (2000a). The unitiy and diversity of executive functions and their contributions to complex "Frontal lobe" tasks: a latent variable analysis. Cogn. Psychol. 41, 49-100. doi: 10.1006/cogp.1999. 0734

Miyake, A., Emerson, M. J., and Friedman, N. P. (2000b). Assessment of executive functions in clinical settings; problems and recommendations. Semin. Speech Lang. 21, 169-183.

Nebes, R. D., Butters, M. A., Mulsant, B. H., Pollock, B. G., Zmuda, M. D., Houck, P. R., et al. (2000). Decreased working memory and processing speed mediate cognitive impairment in geriatric depression. Psychol. Med. 30, 679-691.

O’Brien, J. T., Lloyd, A., McKeith, I., Gholkar, A., and Ferrier, N. (2004). A longitudinal study of hippocampal volume, cortisol levels, and cognition in older depressed subjects. Am. J. Psychiatry 161, 2081-2090. doi: $\quad$ 10.1176/appi.ajp.161. 11.2081

O'Hara, R., Coman, E., and Butters, M. A. (2006). "Late life depression," in Clinical Neuropsychology: A Pocket Handbook for Assessment, 2nd Edn., eds P. G. Snyder, P. D. Nussbaum, and D. Robins (Washington, DC: American Psychological Association), 183-209.

Reitan, R. M., and Wolfson, D. (1993). The HalsteadReitan Neuropsychological Test Battery: Theory and Clinical Interpretation, 2nd Edn., Tucson, AZ: Neuropsychology Press.

Rodriguez-Aranda, C., and Sundet, K. (2006). The frontal hypothesis of cognitive aging: factor structure and age effects on four frontal tests among healthy individuals. J. Genet. Psychol. 167, 269-287. doi: 10.3200/GNTP.167.3. 269-287

Savla, G. N., Twamley, E. W., Thompson, W. K., Delis, D. C., Jeste, D. V., and Palmer, B. W. (2011). Evaluation of the specific executive functioning skills and the processes underlying executive control in schizophrenia. J. Int. Neuropsychol. Soc. 17, 14-23. doi: 10.1017/\$13556177100 01177

Semkovska, M., and McLoughlin, D. M. (2013). Measuring retrograde autobiographical amnesia following electroconvulsive therapy. Historical perspective and current issues. J. ECT 29, 127-133. doi: 10.1097/YCT.0b013e318279c2c9

Sexton, C. E., McDermott, L., Kalu, U. G., Herrmann, L. L., Bradley, K. M., Allan, C. L., et al. (2012). Exploring the pattern and neural correlates of neuropsychological impairment in late-life depression. Psychol. Med. 42, 1195-1202. doi: $\quad 10.1017 /$ S0033291711 002352

Sheehan, D. V., Lecrubier, Y., Sheehan, K. H., Amorim, P., Janavs, J., Weiller, E., et al. (1998). The MINI international neuropsychiatric interview (MINI); the development and validation of a structured diagnostic psychiatric interview for DSM-IV and ICD-10. J. Clin. Psychiatry 59(Suppl. 20), 22-33.

Sheline, Y. I., Barch, D. M., Garcia, K., Gersing, K., Pieper, C., WelshBohmer, K., et al. (2006). Cognitive function in late life depression: relationship to depression severity, cerebrovascular risk factors and processing speed. Biol. Psychiatry 60, 58-65. doi: $\quad$ 10.1016/j.biopsych.2005. 09.019

Steffens, D. C., Otey, E., Alexopoulos, G. S., Butters, M. A., Cuthbert, B., Ganguli, M., et al. (2006). Perspectives on depression, mild cognitive impairment, and cognitive decline. Arch. Gen. Psychiatry 63, 130-138. doi: 10.1001/archpsyc.63. 2.130

Steffens, D. C., and Potter, G. G. (2008). Geriatric depression and cognitive impairment. Psychol. Med. 38, 163-175. doi: 10.1017/S003329170700102X

Snyder, H. R. (2013). Major depressive disorder is associated with broad impairments on neuropsychological measures of executive function: a meta-analysis and review. Psychol. Bull. 139, 81-132. doi: 10.1037/ a0028727

Story, T. J., Potter, G. G., Attix, D. K., Welsh-Bohmer, K. A., and Steffens, D. C. (2008). Neurocognitive correlates of response to treatment in late-life depression. Am. J. Geriatr. Psychiatry 16, 752-759. doi: $\quad 10.1097 / J G P .0 b 013 e 318$ $17 \mathrm{e} 739 \mathrm{a}$

Strauss, E., Sherman, E. M. S., and Spreen, O. (2006). A Compendium of Neuropsychological Tests, 3rd Edn., New York, NY: Oxford University Press.

Stroop, J. R. (1935). Studies of interference in serial verbal reactions. J. Exp. Psychol. 18, 643-666. doi: 10.1037/h0054651 
Sullivan, J. R., Riccio, C. A., and Castillo, C. L. (2009). Concurrent validity of the tower tasks as measures of executive function in adults: a metaanalysis. Appl. Neuropsychol. 16, 62-75. doi: 10.1080/090842808 02644243

Wechsler, D. (2007). Wechsler Abbreviated Scale of Intelligence (WASI). Norwegian Manual Supplement. Stockholm; Pearson Assesment.
Wilkins, C. H., Mathews, J., and Sheline, Y. I. (2009). Late life depression with cognitive impairment: evaluation and treatment. Clin. Interv. Aging 4,

51-57. doi: 10.2147/ CIA.S3154 (1)

Conflict of Interest Statement: The authors declare that the research was conducted in the absence of any commercial or financial relationships that could be construed as a potential conflict of interest.

Received: 18 April 2013; accepted: 10 June 2013; published online: 27 June 2013.

Citation: Dybedal GS, Tanum L, Sundet K, Gaarden TL and Bjølseth TM (2013) Neuropsychological functioning in latelife depression. Front. Psychol. 4:381. doi: 10.3389/fpsyg.2013.00381
This article was submitted to Frontiers in Cognition, a specialty of Frontiers in Psychology.

Copyright (c) 2013 Dybedal, Tanum, Sundet, Gaarden and Bjølseth. This is an open-access article distributed under the terms of the Creative Commons Attribution License, which permits use, distribution and reproduction in other forums, provided the original authors and source are credited and subject to any copyright notices concerning any thirdparty graphics etc. 